\title{
Graphic Representations and Frequency Parameters of Heart Sound Signals
}

\author{
Božo Tomas \\ Faculty of Mechanical Engineering and Computing, University of Mostar, Matice hrvatske bb, 88000, \\ Mostar, Bosnia and Herzegovina \\ Email: bozo.tomas@hteronet.ba \\ Darko Zelenika \\ Faculty of Information Studies, Ljubljanska cesta 31A, 8000, Novo mesto, Slovenia \\ Email: zelenika.darko@gmail.com

\begin{abstract}
Željko Rončević
University Clinical Hospital Mostar, Clinic for Children's Diseases, Cardiology Department, Bijeli brijeg bb, 88000, Mostar, Bosnia and Herzegovina

Email: zroncevic112@gmail.com
\end{abstract}

Received: 24 April 2018; Accepted: 16 May 2018; Published: 08 July 2018

\begin{abstract}
Sounds produced by acoustic activity of the heart are series (sequences) of quasi-periodic events which are repeated throughout life, one period (cycle) of these events lasts less than one second. The advancements in technology have enabled us to create various tools for audio and graphic representations of these events. Physicians, by using such tools, can more accurately determine diagnosis by interpretation of heart sound and/or by visual interpretation of graphic displays of heart sounds. This paper presents frequency parameters and graphic illustrations of heart sound signals for two groups of heart murmurs: innocent Still's murmur and pathologic heart murmur of Ventricular Septal Defect (VSD). Also, on behalf of the frequency analysis of acoustic cardiac signals with Still's murmur was given a medical explanation of cause and origin of Still's murmur.
\end{abstract}

Index Terms-Heart Sound Frequency Parameters, Heart Sound Graphic Representation, Haert Sound Spectrogram, Phonocardiogram (PCG), Still's Murmur, Ventricular Septal Defect (VSD).

\section{INTRODUCTION}

Our body transmits sound messages and „speaks“ about the state of our vital organs (heart, lungs,..). Physicians listen and interpret these sounds. Auscultation is a medical term for listening of internal body sounds and the procedure of listening is mainly done with a stetoscope. That term originates from the Latin word auscultare, which means to listen. The beginning of auscultation started back in 1816 when French physician René Théophile Hyacinthe Laennec
(1781-1826) invented the stethoscope and introduced the term auscultation into medicine [1].

Heart auscultation is very subjective because diagnosis of heart sounds could be interpreted in several ways depending on how a physician interprets the sound. Due to limited opportunities of heart auscultation, it is necessary to help the human ear and make a graphic display of the heart sound. Visual representations of heart sound signals can help physicians to better understand, determine and evaluate heart sound cycle events.

Despite numerous heart sound graphic representations, vast majority physicians do not really use them. One of the most common graphic representations of heart sound signals is phonocardiogram (PCG) (the time display of heart sound amplitudes). Other display of heart sound signals is the heart sound spectrogram which allows better heart sound interpretation, but it is hardly percepted or used by physicians.

With this purpose, authors in [2] introduced a solution for graphic display of heart sounds called HSLs (Heart Sound Lines). Authors believe that graphic display of heart sound signals like this could be a useful tool for the heart sounds interpretation and can assist physicians for a more precise diagnosis of innocent and pathologic murmurs (auscultation-visual diagnosis). The advantage of HSLs graphical display over other methods is in its easier interpretation by their parameters: murmurs color line, numerical value of murmurs index and lines shape.

The paper is organised as follows. In Section II recordings of heart sound signals and Goertzel algorithm are shortly presented. In Section III are shown the spectral compositions (spectral energy distributions) of Still's and VSD's murmur and their frequency parameters. Graphic representations of heart sounds are in Section IV. Section $\mathrm{V}$ concludes the paper with final remarks. 


\section{MATERIALS AND METHODS}

While examining the children in an outpatient clinic by pediatric cardiologists, their heart sounds were recorded with an electronic stethoscope. All children were additionally examined with ultrasound for an accurate diagnosis of congenital heart disease. The recorded heart sounds were classified into three groups: heart sounds without heart murmur - Normal (10 records), heart sounds with physiological Still's innocent murmur - Still (20 records) and heart sounds with pathological murmur associated with congenital heart disease - VSD (20 records).

Heart sounds were recorded with the sampling frequency of $f_{s}=8000 \mathrm{~Hz}$ and resolution of quantization of 16 bits. Further more, in a process of determination of frequency parameters of the murmurs, the complete systolic duration was isolated (by hand using software tool Audacity) from the heart audio files. An energy spectrum of the heart sound data was obtained by applying Goertzel algorithm.

\section{A. Basic Goertzel algorithm}

The algorithm was introduced by Gerald Goertzel (1920-2002) in 1958 [3]. Equation (1) describes the signal flow for the basic Goertzel algorithm as each sample is processed [4]. The signal flow of the algorithm produces an output $y_{0}$ for each processed sample.

$$
y_{0}=x_{0}+y_{1} \times 2 \cos \left(\frac{2 \pi m}{N}\right)-y_{2}
$$

In the equation (1), $y_{0}$ denotes the current output, $x_{0}$ denotes the current input sample, $y_{1}$ denotes the output that is previously processed, $y_{2}$ denotes the second previously processed output, $N$ denotes the size of input block, while $m$ denotes bin number in the frequency domain. Each sample of the input block (of size $N$ ) is processed accrding to the equation (1) and at the end of each block the spectral energy of each frequency bin is computed by the equation (2). This process continues over the next block (of size $N$ ) until the last block is processed.

$$
E=y_{1}^{2}+y_{2}^{2}-2 y_{1} y_{2} \cos \left(\frac{2 \pi m}{N}\right)
$$

In equation (2) $y_{1}$ is the last processed output (iteration $N)$ equation (1) and $y_{2}$ second-last processed output (iteration $N-1$ ).

The advantage of the Goertzel algorithm is that it can process the input data as it arrives. The output value is only needed for the last sample in the block unlike the Fast Fourier Transform (FFT). The FFT has to wait until the entire sample block has arrived. If the number of frequency bins is a lot smaller than $N$, the Goertzel algorithm reduces the data memory which is required significantly. The Goertzel algorithm is more efficient than FFT only when a small number of frequency bins $M$ need to be calculated $\left(M<\log _{2} N\right)$. The motivation for using Goertzel algorithm is in possibility of selection of parameters $\mathrm{m}$ by which we can easily change and adjust frequency resolution and frequency band of analysed signal.

\section{SPECTRAL COMPOSITION AND FREQUENCY PARAMETERS OF HEART MURMURS}

The basic events of a heart sound cycle are first heart sound (S1), second heart sound (S2) and time periods between them. A time between $S 1$ and $S 2$ is called a systole and a time period between $S 2$ and $S 1$ is called a diastole. If there is a sound (noise) which is heard through the systole or diastole, that phenomenon is respectively called systolic heart murmur and diastolic heart murmur.

When murmurs appear, they can last only a small part or entire systole i.e. diastole. The time interval of murmur appearance is very short. At children age, a systolic time interval is around $200 \mathrm{~ms}$ and diastolic time interval is little longer.

Spectral composition of heart sound signals is very useful in detection and heart murmur diagnosis. Majority of authors in their studies mostly used FFT and/or Wavelet Transform for a spectral anlysis of the heart sounds [5-7]. Heart sound spectral analysis with Goertzel algorithm is proposed in [8-9].

In this analysis Goertzel algorithm was applied with the sample block size of 160 samples. When using a sampling frequency of $8 \mathrm{kHz}, 160$ samples $(N=160)$ in discrete time represent the time frame of $20 \mathrm{~ms}$ in realtime. The bin bandwidth frequency is determined by sampling frequency and sample block size $\left(B=f_{s} / N\right)$. The overlapping of bins (frequency resolution) is adjusted by the selection of coefficient $m$ in equations (1) and (2). Figures 1 and 2 illustrate the results calculated by the frequency resolution of $5 \mathrm{~Hz}$.

Frequency parameters of heart murmurs carry information about the health status of patient's heart and these parameters are determined and evaluated in heart sound computational diagnosis. Analysed frequency parameters of heart murmurs (in this article) are:

- Frequency of spectral extremes i.e. frequency on which murmur spectrum has the highest energy (peaks);

- Frequency bandwidth;

- Intensity of spectral energy on resonant frequency (spectral energy of peaks).

\section{A. The spectral compositions of Still's and VSD murmur}

The heart sound graphic images represent heart sound intensity in time and/or frequency besides that time display of heart sound amplitudes does not give information about heart sound frequency and heart sound energy. Display of heart sound spectral energy is a usefull for determination and evaluation of heart murmur. Fig. 1 and Fig. 2 illustrate the distribution of spectral energies of isolated systoles for three typical Still's murmurs (Fig. 1) and for three typical VSD murmurs (Fig. 2). Fig. 1 represents three Still's murmurs (low frequency - Still1, high frequency - Still3 and common - Still2) and Fig. 2 represents three VSD-s (high energy - VSD1, medium energy VSD2 and low energy - VSD3). 


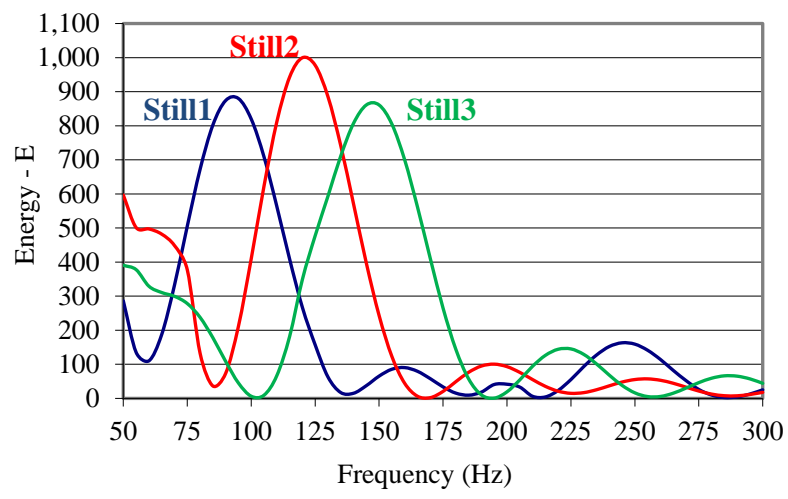

Fig.1. Three typical Still's murmurs

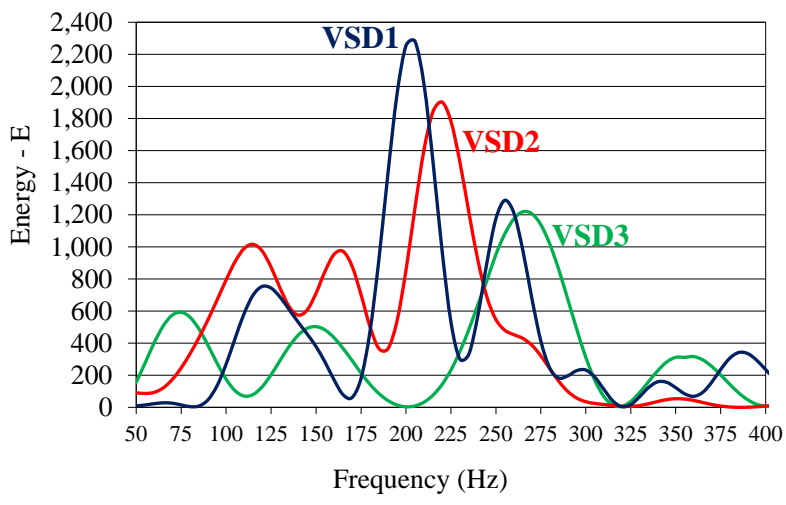

Fig.2. Three typical VSD murmurs

It is clearly visible in Fig. 1 that the information about Still's murmur occurs in a lower frequency bandwith (80$170 \mathrm{~Hz}$ ). It is obvious that the frequency of Still's peak is lower than $200 \mathrm{~Hz}$ while the highest VSD peak has the frequency above $200 \mathrm{~Hz}$.

If we compare spectral compositions of Still's and VSD murmur, information about VSD murmur is in a wider frequency bandwidth and in most cases VSD murmur has a distribution of the spectrum energy in bandwith $(90-300 \mathrm{~Hz})$.

\section{B. Frequency parameters of Still's murmur}

Innocent murmurs are common in children and the most frequent is Still's murmur [10], which occurs and is audible at the beginning of the systole. For every prerecorded heart sound signal, every systole is manually located and secluded for spectral analysis. In Fig. 3 secluded time interval is represented (red rectanglesthree heart sound cycles). Also Fig. 3 shows a PCG display of one Still's murmur before processing (top picture) and after processing by 3M Littmann sound analysis software (bottom picture).

Signal after processing (Low pass filter) has more emphasized murmur and better visual impression. 3M Littmann sound analysis software has three heart sound signal processing option filters (Low pass, High pass and Band pass). Low pass filter is suitable for Still's murmur emphasing and a high pass or band pass filter for VSD murmur.

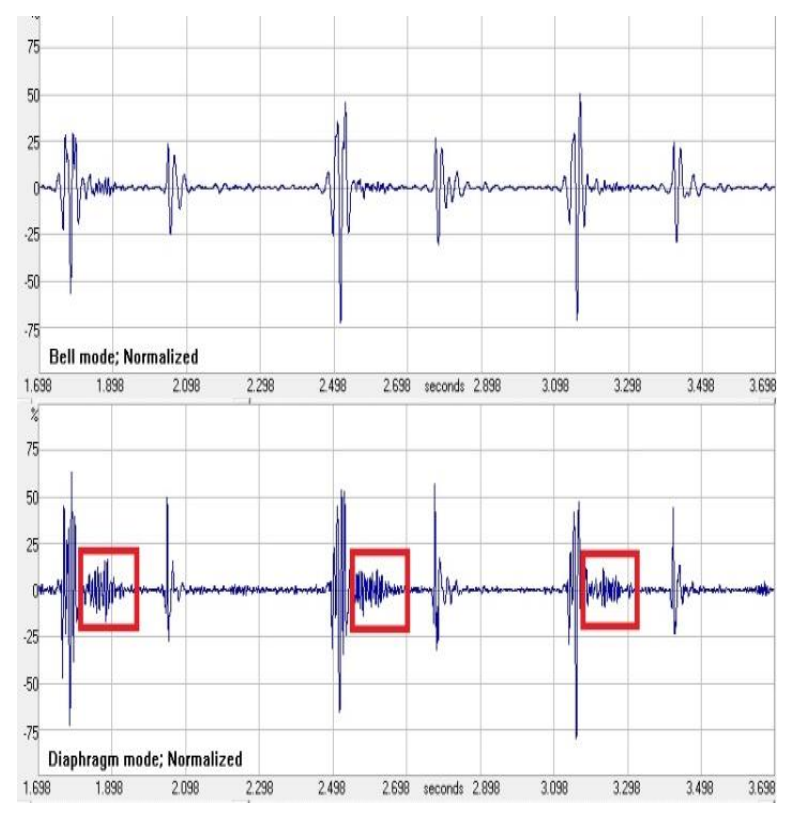

Fig.3. Time interval of Still's murmur

Spectral energy is calculated for the secluded time interval that lasts cca. $100 \mathrm{~ms}$ with frequency resolution of $5 \mathrm{~Hz}$ in time frames (intervals) of $20 \mathrm{~ms}$. The point where Still's murmur has the maximal spectral energy in time interval of $20 \mathrm{~ms}$ is selected as the final position of Still's murmur. That time frame represents the location of Still's murmur and the frequency and the bandwith of Still's murmur are calculated in this time frame. The final position of Still's murmur i.e. the frequency at which it has maximal spectral energy (the peak) is usually in the bandwidth $(B)$ between 110 and $130 \mathrm{~Hz}$. The frequency bandwidth $(B)\left(B=f_{\max }-f_{\min }\right)$ is obtained in a way that the frequency of the final Still's position (the peak) falls in half of strength $f_{\min }$ (left of the top of curve) and $f_{\max }$ (right of the top of curve) [9].

Fig. 4 illustrates the graphic representation of frequency parameters of Still's murmur. It shows that peak of the murmur's spectral energy is at the frequency of $147,5 \mathrm{~Hz}$ on 880 units (values obtained by Goertzel algorithm), $f_{\min }$ is at the frequency around $124 \mathrm{~Hz}$ and $f_{\max }$ is at the frequency around $168 \mathrm{~Hz}$. Therefore, the frequency of this Still's murmur is $147,5 \mathrm{~Hz}$, the bandwidth is cca. $44 \mathrm{~Hz}$ and the spectral energy is 880 .

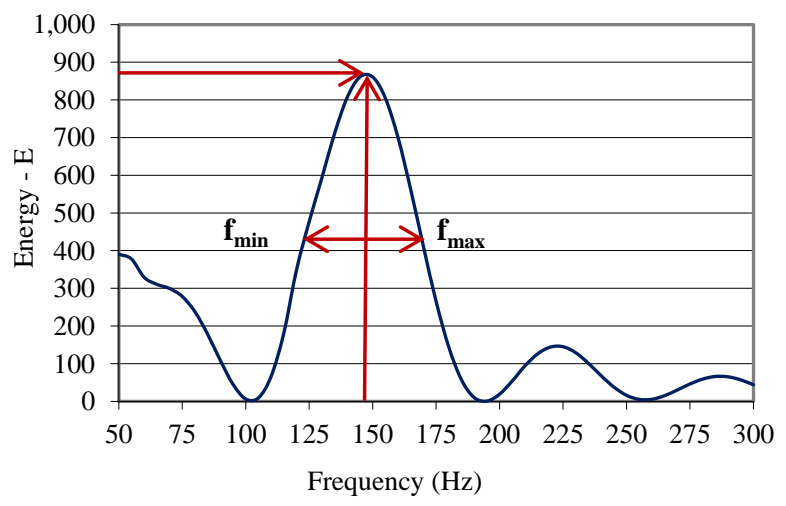

Fig.4. Frequency parameters of Still's murmur 


\section{Frequency parameters of VSD murmur}

VSD murmur is audible in the whole systole. VSD can have a couple of peaks (mostly two or three) which have a slightly lower energy than the uppermost peak. For the VSD with two or more peaks, $f_{\min }$ is to the left of the uppermost peak and $f_{\max }$ is to the right of the uppermost peak. Fig. 5 shows one VSD murmur in the final position (maximal energy of uppermost peak).

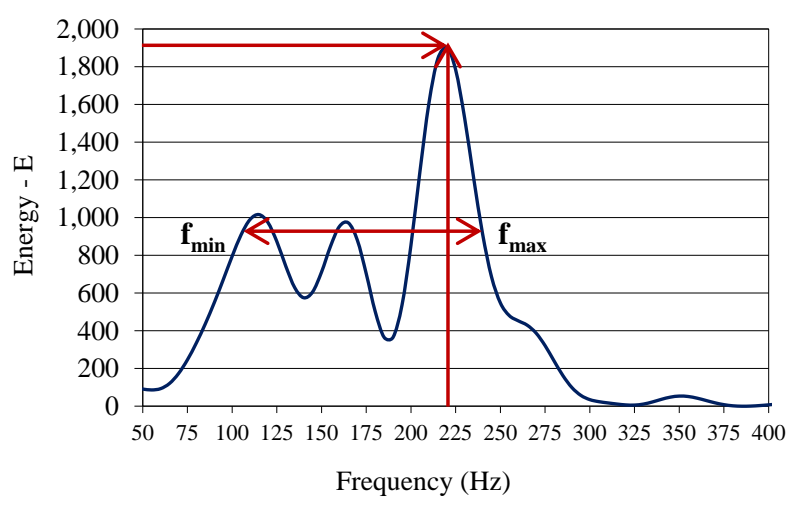

Fig.5. Frequency parameters of VSD murmur

The Fig. 5 shows that the spectral energy of this VSD murmur is at the frequency of $220 \mathrm{~Hz}$ and its energy is 1902,4 units, $f_{\min }$ is at the frequency around $107 \mathrm{~Hz}$ and $f_{\max }$ is at the frequency around $239 \mathrm{~Hz}$. Therefore, the frequency of this murmur is $220 \mathrm{~Hz}$, the frequency bandwidth is $132 \mathrm{~Hz}$ and the spectral energy is 1902,4.

\section{GRAPHIC REPRESENTATION OF HEART SOUND}

The author [11] tested 126 medical students and 20 pediatricians and found that those participants who could play musical instrument or sing in a chorus identified more murmurs correctly than those who had no practical musical skills. A graphic representation of heart sound signal provides a visual image of the heart sound. The PCG shows a change in amplitude of heart sound in time. It is a considerable source of information that can lead by its analysis, to the detection and the identification of several heart abnormalities [12]. Each event in the heart sound cycle (sounds and murmurs) changes the amplitude of PCG base line and physicians can see that change. However, by PCG we can only detect heart sounds and murmurs and show their position and shape in time. A heart sound spectrogram shows the frequency components of heart sound signals and the distribution of spectral energy of heart signals in time. Each event in the heart sound cycle $(S 1, S 2$ as well as murmur if it exists in systole or diastole) has its own spectral energy distribution. The spectral energy of first $\mathrm{S} 1$ and second S2 heart sound is mostly distributed in bandwidth under $100 \mathrm{~Hz}$. Heart sounds $S 1$ and $S 2$ are the loudest events (the highest energy) in cardiac cycle. That is the reason why they have the highest amplitude in PCG representation of heart sounds cycle. Likewise, if a murmur appears in heart sound cycle then each murmur has a unique PCG and spectrogram shape.
Different heart murmurs have different time amplitudes and spectral energy distributions. In this article we graphicly presented only two murmur types (Still and VSD). The spectrogram was created using Matlab. Fig. 6 illustrates graphic representations (PCG and spectrogram) of Still's murmur and Fig. 7 illustrates graphic representations (PCG and spectrogram) of one cardiac signal with VSD murmur.
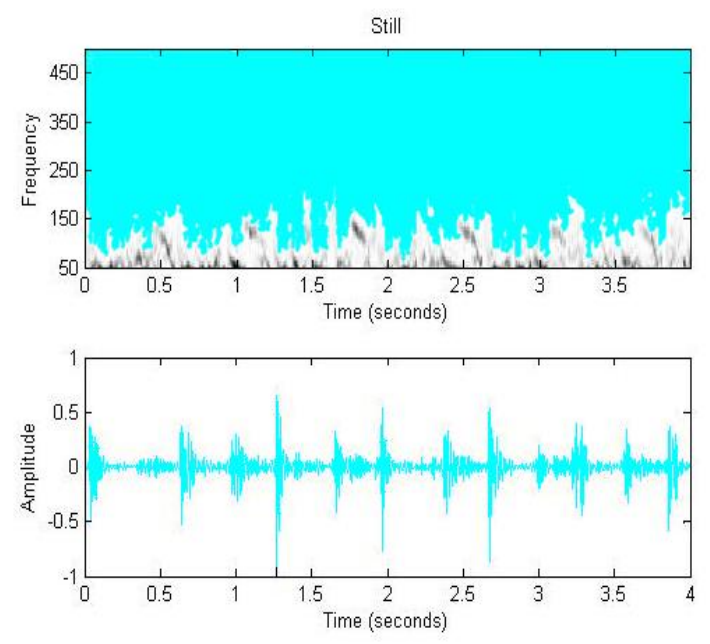

Fig.6. PCG and spectrogram representation of Still's heart murmur

Heart sound signals are mainly unsteady signals in time span. Spectral energy of Still's murmur in Fig. 6 is distributed in bandwidth 100-150 Hz. On PCG representation Still's murmur has a diamond (crescendodecrescendo) shape. That shape is a result of increasing and decreasing of the sound generated by Still's murmur. Cresscendo and decrescendo are expressions taken from music art. Still's murmur is silent at the beginning then it becomes louder in the middle and then declines and stops.

There are no common views on the occurrence of the Still's murmur. The doctors (physicians) haven't yet established reliable genesis of the occurrence of that murmur, answering which heart structures and during which heart developments for the duration of systole that tone is created. Authors' opinion, based on the acoustic analogy, is that this sound can be generated by some string (thread) which vibrates in the appropriate resonator. The hypothesis that the Still's murmur appears during vibrations of cords in the heart is also stated by other authors (physicians) in [13-14]. Cords are thin structures (like threads) within the heart and during the contraction of heart muscles with which they are connected, the cords are vibrating, and at the same time (systole) the ventricles are emptying creating resonator box in which the cords are vibrating and generate sound which we can hear as the Still's murmur. Therefore, alike live instrument, the heart, or more precisely vibrating cord in the heart, starts to play silently, then louder and after maximal loudness starts to appease and stops playing. During diastole, there is no contraction of the heart muscle and no actuation (vibration) of cords. Since cords are not vibrating during diastole, the Still's murmur in diastole is not generated. 

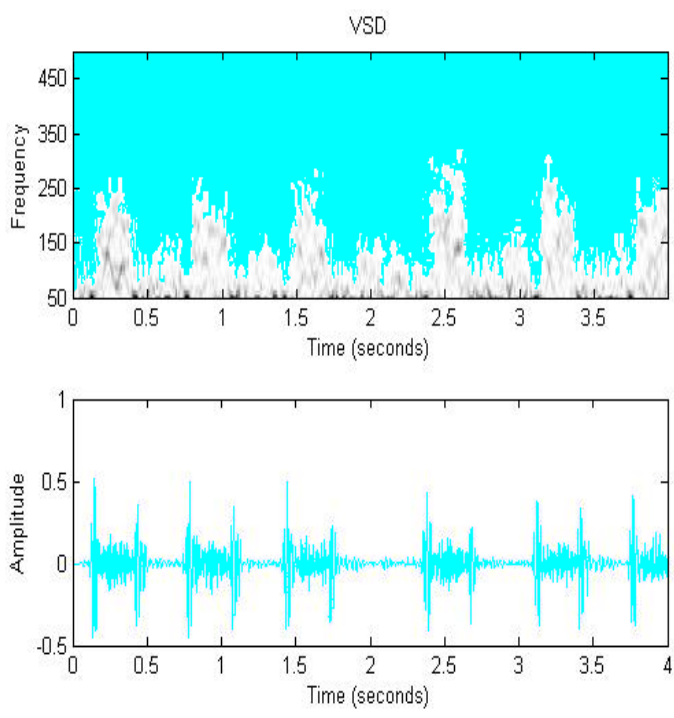

Fig.7. PCG and spectrogram representation of VSD heart murmur

Still murmur is vibratory, musical sound without any evidence of turbulent flow. VSD is harsh systolic murmur of ventricular septal defect (VSD) caused by turbulent blood flow through a defect (,a hole”) in ventricular septum. Ventricular septum separates right and left ventricle. In healthy children and adults septum is intact [8].

In PCG and spectrogram it can be noted that Still and VSD are located in the systole. Heart sounds $S 1$ and $S 2$ have higher amplitudes in PCG display than the amplitudes of Still's and VSD murmur. The spectral energy of Still's murmur is distributed in a narrow frequency bandwidth, which is very close to heart sound bandwidth where the energy of tones $S 1$ and $S 2$ is expressed. In many cases these two bandwidths are overlapping. Therefore, this makes the detection of Still's murmur difficult. This is also the reason and explanation why physicians mostly give a wrong diagnosis by auscultation alone when it comes to Still's murmur.

It is obvious that there is a masking of frequency in Still's murmur and therefore many physicians can't even hear it. The spectral energy of VSD is distributed in a wider frequency bandwidth $80-300 \mathrm{~Hz}$ and it lasts throughout the whole systole. Band of VSD is separated from band of tones $S 1$ and S2. VSD murmur is not masked by tones $S 1$ and $S 2$ so physicians mostly do not have difficulties in recognition of VSD murmur by auscultation technique.

Frequency distribution of heart sounds (S1 and S2) bandwits and heart murmur bandwidth have the best representations on 3D heart sound spectrogram. Fig. 8 illustrates a 3D graphic spectrogram together with PCG for one cardiac signal with Still's murmur and one with VSD murmur.

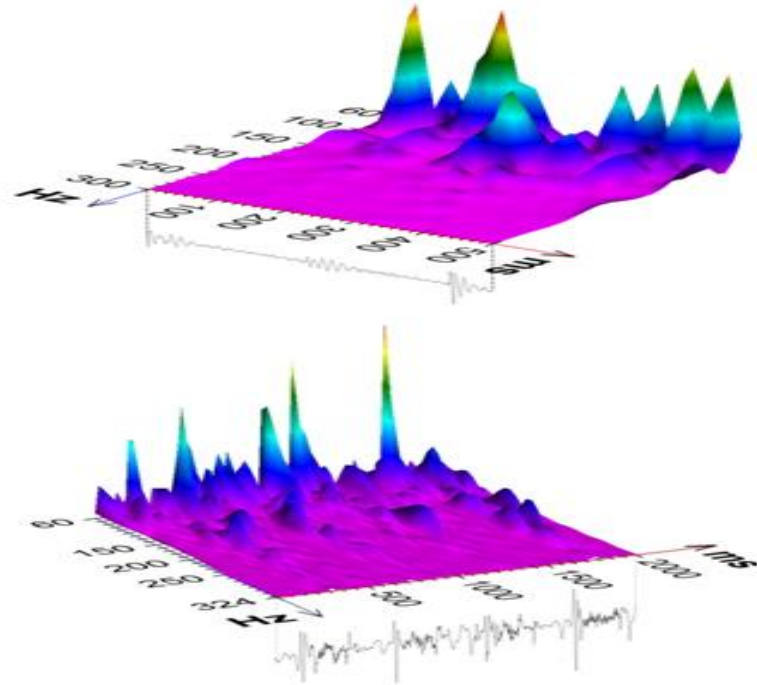

Fig.8. 3D spectrogram with time domain representation of Still's (top picture) and VSD heart murmur (bottom picture)

The top picture shows 0,5 seconds of a spectrogram of one-half cardiac cycle of one heart sound signal with Still's murmur (S1 - Still's murmur in systole - S2). The frequency bandwidth of heart sounds ( $\mathrm{S} 1$ and $\mathrm{S} 2$ ) is shown by hills above base-plane in bandwidth $60-100 \mathrm{~Hz}$ while Still's murmur is shown by one hill of a smaller amplitude than heart sounds in frequency bandwith 100$150 \mathrm{~Hz}$. On 3D representation is visible a small distance between heart sounds and Still's murmur bandwidths. The bottom picture shows 2 seconds of spectrogram of almost four heart sound cycles of one heart sound signal with VSD murmur. Heart sounds bandwidth and VSD murmur bandwidth have a large enough distance.

Generally, by spectral analysis of heart sound signal we can recognize and classify heart murmurs by comparing spectral energy in the defined frequency bandwidth. The graphic displays of Still's and VSD murmur are clearly different, and that is what enables their visual classification. They have different acoustic and frequency parameters and their graphics are different. However, in real medical practice there are many types of murmurs and there are some types of murmurs which have similar frequency parameters with similar graphic displays.

With HSLs graphic representation physicians can easily make murmur classifications (innocent or pathologic) by comparing different lines i.e. their color and value, by comparing values of index murmur and estimating duration of the murmur. HSLs graphic representation shows three pictures: PCG signal on top, heart sounds locations (black line) and murmur evaulation (blue and red lines) on midle and murmur index lines and values on the bottom picture. Fig. 9 illustrates HSLs of one innocent Still heart murmur and Fig. 10 illustrates HSLs of one VSD murmur. 

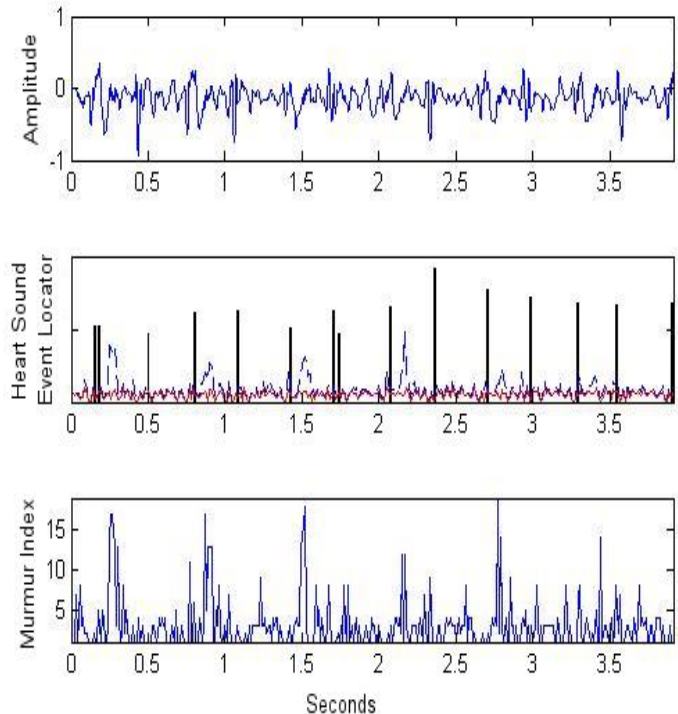

Fig.9. HSLs of Still's heart murmur
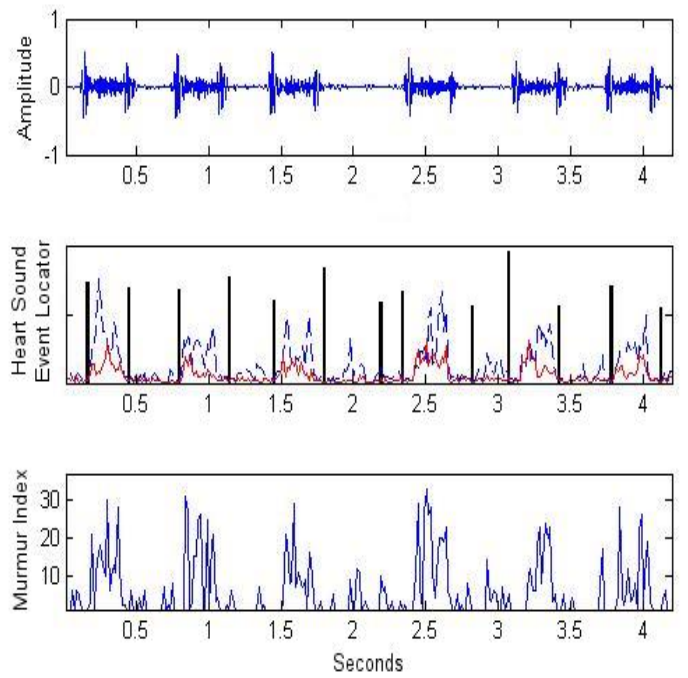

Fig.10. HSLs of VSD heart murmur

Authors created a spectrogram in Matlab and program solution for graphic representation of heart sounds and classification of heart murmurs. The detailed classification procedure of pathologic and innocent heart murmurs by using HSLs tool is described in [2]. HSLs parameters of Still murmur are: blue line, murmur index $<20$ and duration of murmur $<60 \%$. These are also parameters of innocent murmurs. HSLs parameters of VSD murmur are: blue and red lines, murmur index $>20$ and duration of murmur $>60 \%$. These are also parameters of pathologic murmurs. Authors are assuming that HSLs can be used to precisely recognize heart murmur as well as to determine heart rhythm and variation of heart rhythm

The average values of frequency parameters as well as HSLs parameters for 20 Still's murmurs and 20 VSD murmurs are given in Table 1.
Table 1. Parameters of Still's and VSD murmurs

\begin{tabular}{lcc}
\hline Frequency parameters & Still & VSD \\
\hline Average tone frequency $(\mathrm{Hz})$ & 118,75 & 240,82 \\
Average bandwidth $(\mathrm{Hz})$ & 40,75 & 168,1 \\
Average spectral energy of the peak & 998,51 & 1648,65 \\
\hline HSLs parameters & Still & VSD \\
\hline HSLs color lines & blue & Red and \\
Murmur index & $<20$ & $>20$ \\
Murmur duration & $<60 \%$ & $>60 \%$ \\
\hline
\end{tabular}

Average frequency parameters of Still's and VSD murmur are notably different. The frequency of Still's murmur is $118,75 \mathrm{~Hz}$ and of VSD murmur is 240,82 . The frequency bandwidth of Still's murmur is $40,75 \mathrm{~Hz}$ and of VSD murmur 168,1 Hz. The spectral energy of Still's murmur is 998,51 and of VSD murmur is 1648,63. Therefore, all frequency parameters of Still's murmur have lower values than VSD murmur.

Most of pathological murmurs have sounds of higher frequency than innocent. In spectrogram's representation of one Innocent vibratory murmur peak frequency $149 \mathrm{~Hz}$ was recorded [15]. Kudriavtsev et al. [16] demonstrated that Still's murmurs have narrow spectral bandwidth, with this being a significant feature differentiating them from abnormal murmurs. In [17] are presented spectrogram and frequency parameters of three Still's murmurs. Obtained frequency parameters are: peak frequency of first is $102,28 \mathrm{~Hz}$ and bandwidth is $32,1 \mathrm{~Hz}$, peak frequency of second is $124 \mathrm{~Hz}$ and bandwidth is 22 $\mathrm{Hz}$ and peak frequency of third Still's murmur is 127,1 $\mathrm{Hz}$ and bandwidth is $46 \mathrm{~Hz}$. Similar results has also been reported in [18-20].

\section{CONCLUSION}

One of the leading causes of human death is due to cardiovascular diseases. The first step to prevent such diseases is to have an effective method of collecting, monitoring and maintaining the health data of the patient [21]. Biomedical signal processing is an important tool for medical diagnosis and it can help give a medical explanation of cause and origin of medical phenomena. The information such as the temporal location of the heart signals, the number of their internal components, their frequency content, the importance of diastolic breaths and systolic devices can be studied directly on the Phonocardiogram (PCG) signal by the use of signal processing techniques [22].

In this study it is presented that graphic representations of heart sounds can be a reliable assistance tool for heart diagnosis. During heart diagnosis, (classification of heart murmurs) physicians have to recognize the type of murmur. When recognizing heart murmur, both visual and audio, frequency content of a murmur carries murmur information but it is required to know the time interval of a murmur's appearance too (systole or diastole). A spectrogram display of the heart sound gives (enables) 
insight to both domains and provides an additional perspective on the recorded heart sound.

Graphic representations of heart sound signals enable visual murmur displays and their visual classification. Thus, physicians who cannot clearly hear a sound of a heart, with the help of the visual display, will be able to see a sound and then make a diagnosis.

\section{REFERENCES}

[1] Laennec, R. T. H.; De l'Auscultation Médiate ou Traité du Diagnostic des Maladies des Poumons et du Coeur, Paris: Brosson \& Chaudé. The complete title of this book, often referred to as the "Treatise" is: De l'Auscultation Médiate ou Traité du Diagnostic des Maladies des Poumons et du Coeur (On Mediate Auscultation or Treatise on the Diagnosis of the Diseases of the Lungs and Heart, 1819.

[2] Tomas B. and Zelenika D.; Heart Sound Lines - Proposal of a Novel Heart Auscultation Assistant Diagnosis Tool, International Journal of Latest Trends in Engineering and Technology (IJLTET) Vol. 5 Issue 2 March 2015, http://ijltet.org/wp-content/uploads/2015/03/3.pdf

[3] G. Goertzel; An algorithm for the evaluation of finite trigonometric series, American Mathematics Monthly, vol. 65, January 1958, pp. 34-35

[4] Kiser E.; Digital Decoding Simplified Sequential ExactFrequency Goertzel Algorithm, CIRCUIT CELLAR, Issue 182, September 2005, pp. 22-26

[5] Atbi A., Meziani F., Omari T. and Debbal S.M.; Segmentation of Phonocardiograms Signals using the Denoising by Wavelet Transform (DWT), Acad. J. Sci. Res., 1(3): 39-55, 2013.

[6] Djebbari A. and Reguig B.; Short-time Fourier transform analysis of the phonocardiogram signal, The 7th IEEE International Conference on Electronics, Circuits and Systems, pp.844-847, 2002

[7] Debbal S.M. and Bereksi-Reguig F.; Filtering and classification of phonocardiogram signals using wavelet transform, Journal of Medical Engineering \& Technology,vol. 32, no. 1, pp. 53-65, January/February 2008.

[8] Tomas B. and Rončević Ž.; Spectral Analysis of Heart Murmurs in Children by Goertzel Algorithm, The First International Conference on Creative Content Technologies CONTENT 2009, November 15-20, 2009 Athens/Glyfada, Greece, http://circ.ahajournals.org/cgi/reprint/116/14/F79.pdf

[9] Tomas B., Zelenika D., Rončević Ž. and Krtalić A.; Classification of Pathologic and Innocent Heart Murmur Based on Multimedia Presentations of Acoustic Heart Signals, The Third International Conference on Creative Content Technologies CONTENT 2011, September 25-30, 2011 - Rome, Italy ISBN: 978-1-61208-157-1, Pages: 34 to 37 , Archived in the free access ThinkMindTM Digital Library

[10] Still G.F.; Common disorders and diseases of chilhood, 1st ed. London: Frowde, Hodder \& Stoughton, 1909.

[11] Rončević Ž.; Music from the heart-in praise of auscultation, Interview by Keith Barnard, Circulation 2007; 116: 81-2.

[12] A.Choklati, K. Sabri, M. Lahlimi.; Cyclic Analysis of Phonocardiogram Signals, International Journal of Image, Graphics and Signal Processing(IJIGSP), Vol.9, No.10, pp.1-11, 2017.DOI: 10.5815/ijigsp.2017.10.01

[13] Malouf J., Gharzuddine W. and Kutayli F.; A reappraisal of the prevalence and clinical importance of left ventricular false tendons in children and adults, $\mathrm{Br}$ Heart J. 1986;55 (6):587-91.

[14] Kenchaiah S., Benjamin E. J., Evans J. C., Aragam J. and Vasan R. S.; Epidemiology of Left Ventricular False Tendons: Clinical Correlates in the Framingham Heart Study, J Am Soc Echocardiogr. 2009; 22(6): 739-745.

[15] Noponen AL, Lukkarinen S, Angerla A, et al.; Phonospectrografic analysis of heart murmur in children, BMC Pediatrics2007;11:7-23.

[16] Vladimir Kudriavtsev, Kaelber D, Lazbin M, Polyshchuk VV, Roy DL.; New tool to identify Still's murmurs. Pediatric Academic Societies Annual Meeting [http://www.bsignetics.com/papers/PASStillsMurmur.pdf]. 2006 April 29-May 2

[17] Vladimir Kudriavtsev, Vladimir Polyshchuk and Douglas L Roy.; Heart energy signature spectrogram for cardiovascular diagnosis, BioMedical Engineering OnLine 2007, 6:16 doi:10.1186/1475-925X-6-16

[18] Van Oort A, Hopman J, De Boo T, Van Der Werf T, Rohmer J, Daniels O.; The vibratory innocent heart murmur in schoolchildren: A case-control doppler echocardiographic study, Pediatric Cardiol. 1994;15:275281. doi: 10.1007/BF00798120.

[19] Donnerstein RL, Thomssen VS.; Hemodynamic and anatomic factors affecting the frequency content of Still's innocent murmur, Am J Cardiol. 1994;74:508-510. doi: 10.1016/0002-9149(94)90917-2.

[20] Noponen AL, Lukkarinen S, Sikiö K, Angerla A, Sepponen R.; How to recognize the innocent vibratory murmur, Comput Cardiol. 2000;27:561-564.

[21] Sayed Tanvir Alam, Md. Moin Hossain, Mohammad Dehan Rahman, Md. Kafiul Islam, Towards Development of a Low Cost and Portable ECG Monitoring System for Rural/Remote Areas of Bangladesh, International Journal of Image, Graphics and Signal Processing (IJIGSP), Vol.10, No.5, pp. 24-32, 2018.DOI: 10.5815/ijigsp.2018.05.03

[22] A. Choklati, K. Sabri,; Cyclic Analysis of Extra Heart Sounds: Gauss Kernel based Model, International Journal of Image, Graphics and Signal Processing (IJIGSP), Vol.10, No.5, pp. 1-14, 2018.DOI: 10.5815/ijigsp.2018.05.01

\section{Authors' Profiles}

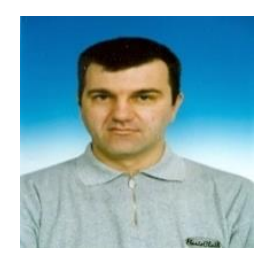

Božo Tomas received Bsc. MSc and Ph.D. degrees at the University of Zagreb, Faculty of Electrical Engineering and Computing in the field of electroacoustics. From 2003. he works at the University of Mostar, Faculty of Mechanical Engineering and Computing (FSR Mostar) as an assistant, 2009. became an Assistant Professor and since 2016. as an Associate Professor. His research areas are speech and biomedical signals (acoustics heart sounds and EKG).

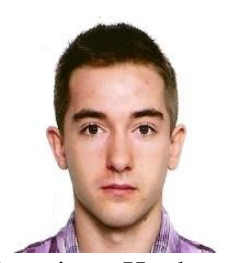

Darko Zelenika received BSc and MSc degrees at the University of Mostar, Faculty of Mechanical Engineering and Computing. He is a $\mathrm{PhD}$ student at the Facutly of Information Studies in Novo mesto (Slovenia) in the field of image processing and machine learning. He has worked as software developer on various 
projects in academic and business fields. His research areas are image processing, machine learning, AI and document analysis.

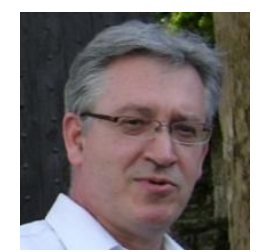

Zeljko Roncevic became MD at University of Sarajevo, Medical School. $\mathrm{He}$ receives $\mathrm{MSc}$ and $\mathrm{PhD}$ degrees at University of Mostar School of Medicine. From 1989 to 2017 he worked at University Hospital Mostar, Bosnia and Herzegovina as a head of Pediatric Cardiology Department. He is Asisstant Profesor of Pediatrics at University of Mostar School of Medicine. His research areas are Pediatric deseases, Pediatric Cardiology, Heart Murmurs in children and Modern technologies in medicine.

How to cite this paper: Božo Tomas, Darko Zelenika, Željko Rončević, " Graphic Representations and Frequency Parameters of Heart Sound Signals ", International Journal of Image, Graphics and Signal Processing(IJIGSP), Vol.10, No.7, pp. 34-41, 2018.DOI: 10.5815/ijigsp.2018.07.04 